\title{
Errores en la comprensión del significado de las letras en tareas algebraicas en estudiantado universitario
}

Errors in understanding the meaning of letters in algebraic assignments by university students

Erros de estudantes universitários na compreensão do significado das letras em tarefas algébricas

\section{Helen Bolaños-González ${ }^{1}$ José Luis Lupiáñez-Gómez ${ }^{1}$}

Received: Set/4/2019 •Accepted: Jan/23/2020 • Published: Jan/31/2021.

\section{Resumen}

Este trabajo tiene como objetivo general analizar, clasificar e interpretar los errores algebraicos que presenta un grupo de 38 estudiantes matriculados en el curso Matemática General de la Universidad Nacional en Costa Rica, que se oferta a diversas carreras. En este estudio, que constituye una réplica de investigación, empleamos un cuestionario centrado en el uso de las letras con diferentes significados, desde etiquetas a variables. Se desarrolló desde el enfoque cuantitativo durante el periodo 2018-2019. Como resultado del análisis se ha detectado que los errores más frecuentes en las respuestas de los ítems responden al uso de la letra como incógnita de valor específico, letra como número generalizado o en el manejo de la letra como variable.

Palabras clave: Errores; dificultades; tareas algebraicas; uso de letras; estudiantes universitarios; álgebra, educación superior; educación matemática.

\section{Abstract}

This investigation has as general objective to analyze, classify and interpret the algebraic errors presented by a group of 38 students enrolled in the General Mathematics course of Universidad Nacional in Costa Rica, which is offered to different majors. In this study, that constitutes a research replica, we use a questionnaire focused on the use of letters with different meanings, from labels to variables. It was developed from the quantitative approach during the 2018-2019 period. As a result of the analysis, it has been detected that the most frequent errors in the answers of the items respond to the use of the letters as unknown specific value, letter as generalized number, or in the use of the letter as variable.

Keywords: Errors; difficulties; algebraic tasks; use of letters; university students; algebra; higher education, Mathematics education.

Helen Bolaños-González \ hbolanos@correo.ugr.es, @ 0000-0002-0406-0831

José Luis Lupiáñez-Gómez \lupi@ugr.es, 0000-0003-3337-6517

1 Departamento de Didáctica de la Matemática, Universidad de Granada, Granada, España. 


\section{Resumo}

Este trabalho tem como objetivo geral analisar, classificar e interpretar os erros algébricos apresentados por um grupo de 38 estudantes matriculados no curso de Matemática Geral oferecido a várias carreiras da Universidade Nacional da Costa Rica. Neste estudo, que constitui uma réplica da pesquisa, utilizamos um questionário focado no uso de letras com significados diferentes, desde rótulos até variáveis. Foi desenvolvido a partir do enfoque quantitativo durante o período 2018-2019. Como resultado da análise, detectou-se que os erros mais frequentes nas respostas dos itens respondem ao uso da letra como incógnita de um valor específico, letra como um número generalizado ou no manejo da letra como uma variável.

Palavras-chave: Erros; dificuldades; tarefas algébricas; uso de letras; estudantes universitários; álgebra, ensino superior; educação matemática.

\section{Introducción}

Los estudios acerca del aprendizaje, la comprensión o los errores que evidencian los estudiantes en su formación universitaria son objeto de indagación sistemática en la educación matemática (Camacho-Machín, Alfonso, Socas \& Depool, 2014; Saglam \& Dost, 2016; Stravou, 2014). Al concretar el contenido matemático en el álgebra, las investigaciones también son frecuentes (Adu-Gyamfi, Bossé, \& Chandler, 2015; García, Segovia, \& Lupiáñez, 2014). La investigación también ha constatado que gran parte de las dificultades de estudiantes de niveles superiores para resolver tareas algebraicas puede provenir de un apego muy sólido a habilidades puramente aritméticas (García, Segovia y Lupiáñez, 2014; Ursini, Escareño, Montes y Trigueros, 2005), al aprendizaje desarrollado en el álgebra escolar en los niveles básicos (Ursini y Trigueros, 2006) e, incluso, a creencias fuertemente asentadas sobre ese contenido matemático (Schlöglmann, 2011). Los estudios con niveles específicos o parcelas del álgebra concretas son muy numerosos.

Godino, Castro, Aké y Wilhelmi (2012) mencionan que una de las dificultades del álgebra es la forma en la que se introduce la aritmética y la matemática elemental en los primeros años de formación, ya que las competencias algebraicas de carácter simbólico son el resultado de un proceso de maduración. Socas (1997) destaca aspectos relevantes en torno a las dificultades, obstáculos y errores que presentan el alumnado en la construcción del álgebra escolar.

Vega, Molina y Castro (2012) describen una investigación realizada con estudiantes de 16 a 18 años, cuyo objetivo fue analizar el sentido estructural que ponen de manifiesto al trabajar con expresiones algebraicas. En su estudio, destacan las dificultades de este grupo de estudiantes cuando resuelven tareas que involucran igualdades notables.

Por otro lado, Pochulu (2009) analiza los patrones de error que presentan estudiantes que inician estudios universitarios, y el provecho que se pueden obtener de esos errores como instrumento de motivación y como punto de partida para exploraciones matemáticas creativas de los alumnos.

Ursini y Trigueros (2006) estudian si la comprensión del concepto de variable mejora cuando los estudiantes cursan matemáticas avanzadas. Estas autoras constatan que el concepto de variable es difícil para los alumnos de distintas edades, y que en los diversos niveles educativos tienen 
dificultades para comprender los usos y aspectos que lo caracterizan. Concluyen que los estudiantes usan con mayor flexibilidad estos aspectos conforme avanzan en los cursos de matemáticas, aunque señalan que su pensamiento algebraico no se desarrolla como se esperaría.

Escalante y Cuesta (2012) realizan un estudio con estudiantes universitarios, con el objetivo de analizar las dificultades en la comprensión del concepto de variable, cuando resuelven problemas, donde se establece una relación del lenguaje algebraico con el lenguaje geométrico, el natural y el aritmético.

García, Segovia y Lupiáñez (2014) investigan los errores más comunes que los alumnos de primer semestre de universidad presentan al operar con los distintos significados que pueden tener las letras en álgebra. Con base en los resultados obtenidos, establecen la clasificación de los estudiantes dentro de alguna de las cuatro categorías de entendimiento en el uso y significado de las letras en álgebra que propone Küchemann (1980).

García (2016) estudia, analiza y caracteriza los errores en los que incurren los estudiantes al resolver tareas algebraicas con el fin de proponer un conjunto de sugerencias dirigidas a implementar estrategias de enseñanza del álgebra. Este estudio se toma como referencia principal para la presente investigación.

En este artículo presentamos un estudio centrado en el proceso de enseñanza y aprendizaje del álgebra a nivel universitario. Específicamente se realiza un análisis como un primer acercamiento en el abordaje del álgebra, tomando como base la aplicación del instrumento The Concepts in Secondary Mathematics and Science Project (CSMS), el cual fue diseñado por Küchemann (1980) para caracterizar la forma de interpretación de los símbolos literales en el tema de álgebra y fue concretado y aplicado por García (2016).

El problema de investigación que abordaremos tiene como objetivo general analizar, clasificar e interpretar los errores algebraicos que presenta un grupo de estudiantes del curso Matemática General de la Universidad Nacional en Costa Rica. De este, se desprenden los siguientes objetivos específicos:

1. Identificar, organizar y caracterizar los errores que presenta un grupo de estudiantes del curso Matemática General, cuando resuelven tareas algebraicas, a partir del instrumento de García (2016).

2. Clasificar a los estudiantes según diferentes niveles de entendimiento en el manejo de las letras.

3. Analizar la dificultad de los ítems del cuestionario en términos de los significados de las letras.

La selección del curso Matemática General se debe a diversos criterios: este es introductorio y requisito para cursos avanzados, lo cual nos permite conocer el nivel del estudiante al ingresar a la educación superior. Según Castillo, Gamboa e Hidalgo (2018), el porcentaje de aprobación de dicho curso es relativamente bajo, así como los porcentajes de deserción y reprobación que también son preocupantes. En este contexto, es importante reflexionar por qué se dan estos resultados en el curso, lo que nos lleva a cuestionar el aprendizaje que los estudiantes traen desde secundaria en el álgebra, pues se considera su base. Además, también resulta importante analizar si los estudiantes poseen las habilidades y destrezas matemáticas necesarias para el desarrollo completo de su formación universitaria. 
Aunado a lo anterior, Castillo-Sánchez, Gamboa-Araya e Hidalgo-Mora (2020) agregan que uno de los motivos para la reprobación en dicho curso es la deficiencia en conocimientos previos, así mismo aseguran que uno de los factores para la reprobación es el bajo rendimiento académico en el primer examen parcial, en este se evalúa el tema de algebra y vuelve a salir como factor de la reprobación la deficiencia en conocimientos previos. Por otra parte, Zamora-Araya (2020) realiza un estudio en un contexto muy similar, la educación secundaria, en el cual obtiene que la confianza en sí mismos, al aprender matemática, incide en el rendimiento académico de los estudiantes.

Esta investigación forma parte de un estudio más amplio (Bolaños, 2019), que replica en este contexto costarricense el trabajo de García (2016), realizado con estudiantes universitarios mexicanos.

\section{Marco teórico}

Dado el propósito de este trabajo de analizar, clasificar e interpretar los errores algebraicos que presenta un grupo de estudiantes, consideramos que los elementos que fundamentan el marco teórico de la investigación son: la concepción del álgebra, la noción de error algebraico, y la complejidad asociada a los ejercicios algebraicos.

\section{La concepción del álgebra}

El estudio del álgebra comprende el desarrollo de un pensamiento algebraico que tiene un amplio campo de aplicación en matemáticas. En esta disciplina, el álgebra es una herramienta para la resolución de problemas y la modelización de situaciones, que incluye desde plantear una ecuación hasta modelizar mediante funciones. El álgebra es un lenguaje para comunicar ideas matemáticas, expresar generalizaciones por medio de los símbolos y, además, es una herramienta para resolver problemas y diseñar modelos matemáticos (Serres, 2011).

Vergel (2015) define el pensamiento algebraico como una forma particular de reflexionar matemáticamente y destaca que, desde las consideraciones filosóficas, ese pensamiento involucra un conjunto de procesos corporizados de acción y de reflexión constituidos histórica y culturalmente.

De acuerdo con Godino y Font (2003), el razonamiento algebraico implica representar, generalizar, y formalizar patrones y regularidades, donde el uso de símbolos y expresiones literales permite progresar en un uso adecuado del lenguaje y del simbolismo necesario para comunicar el pensamiento algebraico.

Ursini y Trigueros (2006) agregan que se debe ayudar al estudiantado a desarrollar el pensamiento algebraico, pero hay que darles oportunidades para reflexionar en los distintos tipos de problemas, de manera que se logre profundizar en la comprensión de las variables hasta llegar a utilizarlas de manera autónoma.

La concepción del álgebra se relaciona con la aritmética y la aritmética generalizada, lo cual presenta dificultades de comprensión respecto a los cambios en el significado de los símbolos entre la aritmética y el álgebra (Serres, 2011).

Küchemann (1980) identifica seis formas de interpretar los símbolos literales. La letra evaluada se entiende como la letra a la que se le asigna un valor numérico. La letra ignorada es la letra no utilizada o aquella cuya existencia es reconocida, pero a la que no se le atribuye ningún significado. A la letra como objeto se la considera como una abreviatura del nombre de un objeto o 
incluso como a un objeto en sí mismo. La letra como incógnita especifica representa un número particular, pero desconocido para que los estudiantes puedan operar directamente sobre ella. La letra como número generalizado, en este caso es vista como una incógnita que puede tomar varios valores. Finalmente, la letra como variable representa un rango de valores no especificados, mediante una relación sistemática entre dos conjuntos de valores de este tipo.

Para ejemplificar las formas de interpretar los símbolos literales en la siguiente figura se muestra los ítems 7 a y $7 \mathrm{~b}$ donde se usa la letra como letra evaluada, el ítem 7c hace uso de las letras como objeto e implica el manejo de las letras como valores desconocidos, finalmente el ítem $7 \mathrm{~d}$ hace uso de las letras como número generalizado.

En su estudio, Küchemann (1980) logra identificar estos usos de letras en las actuaciones de los sujetos y los caracteriza en cuatro niveles de entendimiento en relación con el significado y uso de estas.

- $\quad$ Nivel 1: En el primer nivel, los estudiantes son capaces de resolver ejercicios numéricos o que tienen una estructura algebraica simple, por ejemplo, sustituciones numéricas directas en expresiones algebraicas en donde las letras no tiene coeficiente, multiplicación de datos numéricos, simplificación de términos semejantes que involucran una sola letra como incógnita. Los estudiantes en este nivel deben ser capaces de resolver el ítem mediante el uso de letra como objeto o sin hacer uso de la letra.

- $\quad$ Nivel 2: Este nivel es muy similar al anterior; la diferencia consiste en que el estudiante es capaz de resolver ejercicios un poco más complejos, con una estructura aritmética mayor. En este nivel existe una mayor disposición para aceptar respuestas incompletas o ambiguas. Los estudiantes podrían resolver los ejercicios mediante el uso de las letras como letra evaluada, letra como objeto y letra ignorada.

- $\quad$ Nivel 3: En este nivel los estudiantes deben resolver ejercicios que presentan una estructura algebraica simple mediante la interpretación de las letras como incógnitas de valor específico, números generalizados y variables.

- $\quad$ Nivel 4: El estudiante en este nivel debe resolver ejercicios más abstractos y con una estructura compleja. Se requiere como mínimo que las letras

7. ¿Cuáles son las áreas de estas figuras?

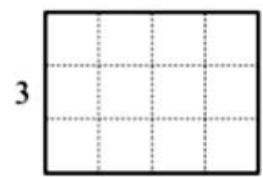

4

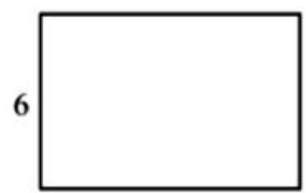

10

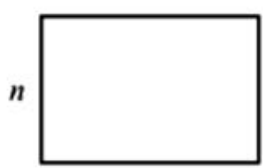

$m$

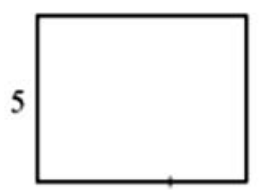

2
$7 a \underline{A=12}$
$7 b \quad A=60$
7c $\mathrm{A}=n \cdot m$
$7 d \quad A=5(e+2)$

Figura 1. Ejemplo del uso de las letras.

Fuente propia de la investigación. 
sean consideradas como incógnitas específicas, pero donde hay una tendencia a tratar las letras como objetos. El estudiante debe ser capaz de distinguir que las letras no son etiquetas de las características propias de los objetos y argumentar que la expresión formulada puede representar distintos valores. Además, debe poder expresar y justificar la respuesta de manera generalizada y no limitarse a la evaluación de solo algunos valores numéricos que satisfagan la expresión.

Para ejemplificar los niveles retomaremos el ejercicio de la Figura 1, los ítems 7 a y $7 \mathrm{~b}$ son categorizados como nivel I, esto, debido a que son sustituciones directas; por otra parte, el ítem 7c se ubica en el nivel II, ya que el estudiante debe ser capaz de resolver un ejercicio con mayor nivel de dificultad respecto al nivel anterior. Finalmente, el ítem $7 \mathrm{~d}$ se ubica en el nivel IV, esto se debe a que el estudiante debe poder expresar y justificar la respuesta de manera generalizada.

Para el logro de los objetivos de la presente investigación, replicamos el trabajo de García (2016), basado en el modelo de Küchemann (1980).

\section{La noción de error algebraico y la complejidad asociada a los ejerci- cios algebraicos}

Escalante y Cuesta (2012) analizan la comprensión de los diferentes aspectos que caracterizan la variable y su relación funcional en situaciones contextualizadas. Estos autores mencionan que se presenta complejidad para trasladar las ideas expresadas en lenguaje natural al lenguaje algebraico. Además, constatan la existencia de dificultades para generalizar los resultados de operaciones aritméticas y para manipular operaciones algebraicas, lo cual puede emerger del contexto de representación de los problemas que se le plantean al estudiante.

De acuerdo con Godino, Castro, Aké y Wilhelmi (2012), el álgebra puede entenderse como un lenguaje simbólico, que se orienta básicamente a la resolución de ecuaciones y al estudio de los polinomios, sin continuidad con los temas de aritmética, medida y geometría tratados en niveles inferiores. Según estos autores, el álgebra aparece de manera abrupta en muchas ocasiones en el proceso educativo, $\mathrm{y}$ eso puede ocasionar distorsiones en la formación matemática de estudiantes, pues no disponen de un proceso de maduración de los conceptos algebraicos.

Escalante y Cuesta (2012) mencionan que la incomprensión del concepto de variable se mantiene igual desde la secundaria hasta la universidad; los estudiantes continúan evitando cualquier acercamiento algebraico y retornan a procedimientos aritméticos, en especial cuando el problema implica cierto nivel de razonamiento, ya que esto les implica un grado de dificultades mayor. Estos autores destacan que el estudiante que logra realizar una lectura analítica de los enunciados verbales, y puede trabajar la traducción entre los lenguajes natural, aritmético y geométrico al lenguaje algebraico, ha desarrollado con solidez su pensamiento algebraico y esto le permite comprender el concepto de variable, sus diferentes aspectos y usos.

Socas (1997) presenta una reflexión general acerca del origen de las dificultades que presentan los estudiantes en el aprendizaje de las matemáticas y las clasifican en cinco categorías. Las dos primeras tienen relación con la disciplina: la complejidad de los objetos matemáticos por un lado y los procesos de pensamiento matemático 
por otro. La tercera categoría se centra en el proceso de enseñanza desarrollado para el aprendizaje de las matemáticas, y tiene relación con los materiales curriculares, los recursos y los estilos de enseñanza. $\mathrm{La}$ cuarta categoría se asocia al proceso del desarrollo cognitivo del estudiante, en el cual se toman en consideración varios enfoques de aprendizaje. Finalmente, existe un grupo de dificultades asociadas a actitudes afectivas y emocionales hacia la matemática, las cuales se vinculan con la ansiedad y el miedo hacia la asignatura.

Esta revisión confirma que existen varias causas que pueden llegar a generar dificultades en el proceso de aprendizaje del álgebra. El tener información sobre la naturaleza de los procesos de aprendizaje y del conocimiento del desarrollo intelectual de estudiantes permite conocer el nivel de dificultad que se les presenta (Socas, 1997).

Las dificultades pueden abordarse desde varias perspectivas: el desarrollo cognitivo de los alumnos, el propio currículo de matemáticas y los métodos de enseñanza. Socas (1997) señala que las dificultades se conectan en la práctica en forma de obstáculo y se manifiestan en el error del alumno. Además, agrega que el error se da por un esquema cognitivo inadecuado.

Mulhern (1989) menciona que en muchas ocasiones el estudiante desarrolla cierta resistencia al dejar de cometer el error: es decir, la corrección de los errores implica, en ocasiones, corregir un conocimiento aprendido erróneamente. Además, agrega que los errores pueden generarse por una comprensión equivocada, que el estudiante considera correcta o errores al azar, que reflejan falta de cuidado. Es decir, cuando un contenido matemático no es significativo para el estudiante y carece de sentido para él, en ocasiones, se puede evidenciar en los errores que presenta el estudiante al resolver ejercicios matemáticos.

Socas (1997) plantea que los errores en el aprendizaje de las matemáticas tienen origen en tres ejes; en su obstáculo, en una ausencia de significado y en actitudes afectivas y emocionales. Asimismo, señala, que algunos errores son producto de la experiencia previa y del desarrollo interno de esas experiencias del estudiante.

Según Rach, Ufer y Heinze (2013), los errores pueden considerarse como oportunidades de aprendizaje, aunque no está claro qué aspectos de la instrucción matemática son relevantes para convertir esto en algo beneficioso, pero sí resalta este como un factor importante en los procesos de enseñanza y aprendizaje que los docentes deben considerar en el planeamiento de la clase.

Este marco de referencia sobre el álgebra escolar y los errores en su aprendizaje nos permiten fundamentar el trabajo de investigación y orientar el análisis de los resultados.

\section{Metodología}

El presente estudio constituye una réplica de investigación desde la perspectiva metodológica. Esta metodología se concibe como:

Un mecanismo de verificación de experiencias empíricas y de exploración de conocimiento nuevo para enriquecer los abordajes de una problemática particular. Así, la realización de réplicas no es solo un proceso de sustitución a partir de un examen conceptual y metodológico que adapta o reorganiza diseños originales a un contexto particular, sino que también un espacio de aprendizaje y formación científica para anclar al estudiante al proceso investigativo. (Ordoñez, 2014, p. 10) 
El trabajo de García (2016) nos permite realizar dicho estudio en el contexto costarricense, llevando a cabo la aplicación del instrumento propuesto originalmente por Küchemann (1980) y aplicado por García para la categorización de los distintos usos de las letras.

La investigación se aborda desde el enfoque cuantitativo, lo cual permite recoger los datos a partir del instrumento de evaluación para conocer el rendimiento algebraico en un grupo de estudiantes universitarios y detectar el tipo de error que presentan cuando se enfrentan a la resolución de tareas algebraicas. Esto hace que nuestro estudio sea de naturaleza diagnóstico-descriptiva (Johnson y Christensen, 2008).

\section{Muestra}

La selección de la muestra es a conveniencia de la investigación, ya que una de las investigadoras tiene una importante experiencia impartiendo el curso de Matemática General en la Universidad Nacional de Costa Rica. En el curso 2018-2019 se trabajó con 38 estudiantes que cursan varias carreras, como las licenciaturas en Economía, Administración, Ingeniería en Agronomía y Biología, y las diplomaturas en Ciencias Geográficas, Cartografía Digital, Comercio y Negocios Internacionales e Ingeniería en Gestión Ambiental.

La muestra que se le aplica el instrumento son 22 hombres y 16 mujeres. En su mayoría son de primer ingreso.

El curso de Matemática General (MAT001) se imparte en el primer año, este es introductorio y requisito para cursos avanzados. Este se describe de la siguiente manera.

En este curso se estudian los conceptos fundamentales del álgebra, funciones, ecuaciones y trigonometría para que el estudiante pueda aplicarlos en su carrera. Además, se prepara al estudiante para que sea capaz de analizar, interpretar y resolver problemas de aplicación de la Matemática, con la finalidad de que desarrolle las habilidades y destrezas necesarias para enfrentar con éxito su desempeño profesional. (Escuela de Matemática, 2017b, p. 1)

Es importante aclarar que la población que se consideró en el estudio de García (2016), realizado en México (194 estudiantes), es superior a la población de Costa Rica (38 estudiantes). Sin embargo, el estudio se realiza en el mismo nivel educativo.

Las edades son similares en ambos estudios, en el caso de México las edades van desde los 17 hasta los 29 años, la más frecuente entre 17 y 19 años. En Costa Rica las edades de los estudiantes oscilan entre 17 y 24 años, las más frecuentes entre los 19 y los 20 años.

\section{Instrumento}

El instrumento utilizado es tomado del proyecto The Concepts in Secondary Mathematics and Science Project (CSMS), que se basa en la categorización de los distintos usos de las letras propuestos por Küchemann (1980).

Se trata de un cuestionario diseñado por Küchemann, Brown y Blaskeley (Küchemann, 1980, p. 16), el cual está conformado por 22 ejercicios, algunos de estos ejercicios se subdividen en ítems, en total se cuentan con 53 ítems que tratan contenidos algebraicos cómo: sustitución formal, simplificación, generalización y formulación, interpretación y solución de ecuaciones. Es importante aclarar que dichos temas son conocimiento adquirido en secundaria en el contexto costarricense. 
Cada ejercicio planteado en dicho instrumento está en función de la respuesta esperada, es decir, el estudiante tenía que interpretar las letras en cada ítem como valores evaluados, objetos, incógnitas con valor específico, números generalizados y variables. La evaluación del instrumento fue organizada de acuerdo con los niveles de entendimiento en el manejo de las letras propuesto por el autor. García (2016) aplicó este cuestionario con una muestra de 194 estudiantes del Centro Universitario de la Costa Sur, México.

\section{Análisis y resultados}

La información recolectada con el instrumento se codificó y se organizó de manera sistemática en una base de datos, resaltando algunos criterios de interés y utilizando fórmulas condicionales para contabilizar algunos ítems.

Los resultados principales de dicha investigación se describen a partir de la complejidad de los ítems, agrupada por los niveles de entendimiento propuestos por Küchemann y considerados por García (2016). La complejidad es medida según el porcentaje de aciertos y errores. Además, se analiza el rendimiento de los estudiantes por el nivel de entendimiento.

Tabla 1

Cantidad de estudiantes por nivel de entendimiento

\begin{tabular}{lc}
\hline & Cantidad de estudiantes \\
\hline Nivel I & 6 \\
Nivel II & 11 \\
Nivel III & 20 \\
Nivel IV & 1 \\
\hline Total & 38 \\
\hline
\end{tabular}

Nota: Fuente propia de la investigación.
Se puede evidenciar que la mayoría de estudiantes participantes se ubica en el nivel III de entendimiento. Es importante mencionar que los temas que se abordaron en el instrumento eran conocimiento de secundaria, lo cual podría suponer que la población participante debería lograr resolver los ítems planteados en el instrumento sin ningún problema. Sin embargo, solo 1 estudiante logra ubicarse en el nivel más alto de entendimiento. Esto quiere decir que demuestra un adecuado dominio en la comprensión y manejo del uso y significado de las letras en diferentes tareas algebraicas.

En la revisión de ítems se han evidenciado errores en ítems donde se presentan letras distintas en la expresión, lo que evidencia un mal manejo de las letras algebraicas por parte del estudiante. En general no logra justificar o expresar de manera generalizada su respuesta y esto ha representado una complejidad para la población participante.

Los ítems del instrumento aplicado que presentaron mayor complejidad para los estudiantes corresponden a los ejercicios de los niveles de entendimiento más altos. Estos son los que están relacionados con el manejo de las letras como números generalizados y como variable, donde se requiere, como mínimo, tratar las letras como objeto.

En el siguiente ejemplo se muestra el ítem con mayor porcentaje de errores, pues la mayoría de la población participante no fue capaz de resolverlo correctamente (Figura 2). En este caso, un estudiante aplica incorrectamente la fórmula del cubo de una suma. 
21.

Si esta ecuación $\longrightarrow$

es verdadera cuando $x=6$,

$(x+1)^{3}+x=349$

Entonces

¿Qué valor de $x$

hará esta ecuación $\longrightarrow$

verdadera?

$x=\frac{87}{20} \ldots$

$7.5 x+1+5 x=(5 x+1)^{3}+5 x=349$

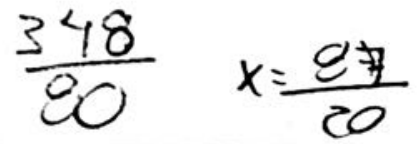

Figura 2. Ejemplo de error por uso de la letra como incógnita de valor desconocido.

Fuente propia de la investigación.

Circunstancias similares se encontraron en el estudio realizado por García (2016); este ítem obtiene un $4.1 \%$ de respuestas correctas, lo que equivale 8 aciertos de los 194 participantes.

En nuestro estudio este ítem de la figura 1 obtiene el número más alto de respuestas en blanco. Esto puede significar que, en general, el estudiante no tiene el conocimiento suficiente para realizar operaciones algebraicas ni para trabajar con números racionales, ni sabe cómo aplicar las reglas algebraicas.

Otro ejemplo de error frecuente es el ítem 3 que evalúa las competencias en el manejo de las letras como números generalizados, y en el que se ha obtenido un 78.95 $\%$ de respuestas incorrectas. La mayoría de estudiantes logran indicar que la expresión que representa el más grande es $2 n$; sin embargo, no es capaz de justificar apropiadamente su respuesta, tal y como muestra la figura 3. El estudiante tiende a resolver mentalmente sin razonar si el procedimiento o el resultado son correctos.

También se ha detectado que el estudiante recurre a procedimientos aritméticos para interpretar problemas. En el siguiente ejemplo se muestra el ítem 22 , donde el estudiante no logra expresar algebraicamente el planteamiento del enunciado (Figura 4), pero sí resuelve el problema dando la respuesta a partir de procedimientos aritméticos.

También se evidencia la tendencia de recurrir al procedimiento aritmético, por ejemplo, en el ítem 17 (figura 5). Se ha comprobado que el estudiante es capaz de resolver el ítem $17 b$ recurriendo a la aritmética, pero que es incapaz de plantear una ecuación correcta que represente el planteamiento del problema.

\section{3. ¿Cuál es el más grande $2 n \circ n+2$ ? $\quad \ldots .$. ...}

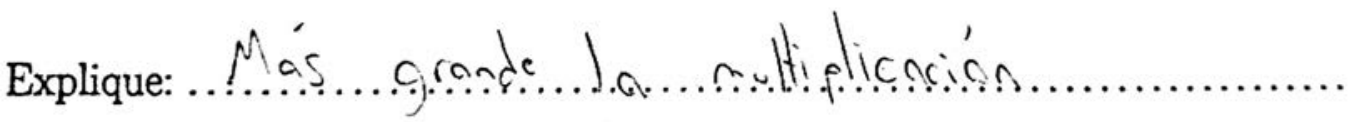

Figura 3. Ejemplo de error por uso de la letra como objeto.

Fuente propia de la investigación. 
22. Los lápices azules cuestan 500 colones cada uno y los lápices rojos cuestan 600 colones cada uno. Si compro algunos lápices azules y algunos rojos y en total me cuesta 9000 colones.

Si $a$ es el número de lápices azules comprados, y si $r$ es el números de lápices rojos comprados, ¿Qué puede

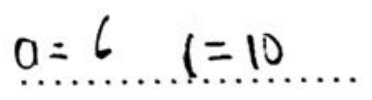
escribir de $a$ y $r$ ?

Figura 4. Ejemplo de error por uso de la letra como objeto. Fuente propia de la investigación.

17. El salario base de María es de 20000 colones por día.

Ella también recibe otro pago de 2000 por cada hora extra que trabaja.

Si $h$ representa el número de horas extra que ella trabaja, y $p$ representa su paga total por día (en $S$ ) escribir una ecuación que relacione $p$ y $h$ :

¿Cuál sería la paga total del día si ella trabajo 4 horas extra?

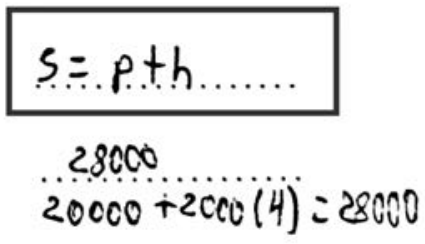

Figura 5. Primer ejemplo de error por uso de la letra como objeto (etiqueta).

Fuente propia de la investigación.

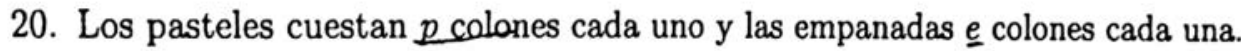

Si compro 4 pasteles y 3 empanadas,

¿Qué significa

$4 p+3 e ?$

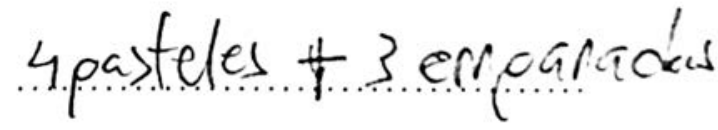

Figura 6. Segundo ejemplo de error por uso de la letra como objeto (etiqueta).

Fuente propia de la investigación.

Con frecuencia se ha detectado también el error en el manejo de letra como objeto. Existe por parte de los estudiantes una fuerte tendencia a relacionar las letras como etiquetas en las que sustituyen el significado abstracto de las letras por algo más concreto.

Al igual que el ejemplo anterior, en la Figura 6 el estudiante recurre a las etiquetas para expresar una situación específica en matemáticas a través del álgebra.

Estos ítems demandan un cierto razonamiento algebraico, debido al grado de complejidad que representa la tarea. En nuestro caso, ha sido evidente que al estudiante universitario aún le falta madurez algebraica. De acuerdo con Escalante y Cuesta (2012), es importante la lectura analítica de los enunciados verbales, para 
8.

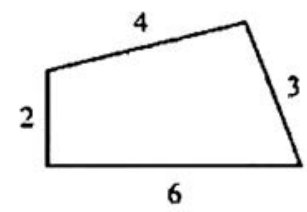

El perímetro de esta figura es igual a $6+3+4+2$, que es igual a 15

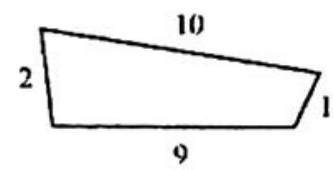

Calcular el perímetro de esta figura. $P=\ldots 22 \ldots \ldots$

Figura 7. Ejemplo del uso de la letra es como objeto.

Fuente propia de la investigación.

permitir reflexionar y traducir los lenguajes natural, aritmético y geométrico al lenguaje algebraico.

Por otra parte, el ítem de mayor número de aciertos con $98.5 \%$ en el estudio realizado por García (2016), similar al contexto costarricense obtiene un alto porcentaje de respuestas correctas, se muestra en la Figura 7. En este caso, los conocimientos requeridos para su solución son operaciones aritméticas como la suma, sustituciones simples y el concepto geométrico de perímetro. El ítem consistía en sumar la longitud de los lados de la figura geométrica para obtener el perímetro.

De manera global, al analizar los 38 cuestionarios, se obtiene que el ítem de mayor número de aciertos obtuvo un $100 \%$ de respuestas correctas (Figura 7), mientras que el ítem de menor número de aciertos fue de $10.53 \%$ lo que equivale a 4 respuestas correctas (Figura 1). Además, el promedio de respuestas correcta por ítem fue de 25 aciertos.

Los errores más frecuentes encontrados en las respuestas de los ítems responden al uso de la letra como incógnita de valor específico, letra como número generalizado o en el manejo de la letra como variable. Estos usos requieren un mayor nivel cognitivo y este resultado también ha sido evidente en la investigación de García (2016).

A continuación, se describen algunas de las situaciones que han conllevado al error algebraico, a partir del análisis de las respuestas del cuestionario aplicado a los estudiantes.

Se refleja un notable desempeño en una matemática memorística, mediante la cual se recuerdan fórmulas, pero se tienen visibles dificultades para aplicarlas. Esto se ha presentado, por ejemplo, en los ítems donde se pide calcular el área o el perímetro donde el estudiante no logra identificar correctamente datos necesarios para aplicar determinadas fórmulas.

Por otro lado, cuando el estudiante se enfrenta a tareas matemáticas recurre a los conocimientos aritméticos que sí domina, pero no logra expresar en términos algebraicos lo que se le pide en el ejercicio. También se evidencia con frecuencia que el estudiante aplica operaciones aritméticas incorrectas o da respuestas incompletas.

Al mismo tiempo, se ha comprobado que el estudiante no es capaz de reflexionar su respuesta, con lo cual evidencia carencia de habilidades propias del pensamiento matemático. Esto se deriva del análisis de los ítems donde el estudiante comete errores 
13. $a+3 a$ puede escribirse de manera más sencilla como $4 a$

Escribe las siguientes expresiones más sencillas, si es posible:

$$
\begin{aligned}
2 a+5 a & =13 a \underline{7 a} \\
2 a+5 b & =13 b \underline{2 a+5 b} \\
(a+b)+a & =13 c \underline{2 a+b} \\
2 a+5 a+a & =13 d \underline{8 a} \\
(a-b)+b & =13 e \underline{a}
\end{aligned}
$$

$$
\begin{aligned}
3 a-(b+a) & =13 f \underline{2 a-b} \\
a+4+a-4 & =13 g \underline{2 a} \\
3 a-b+a & =13 h \underline{4 a-b} \\
(a+b)+(a-b) & =13 i \underline{2 a}
\end{aligned}
$$

Figura 8. Ítem 13 h.

Fuente propia de la investigación.

16. ¿Qué puede decir sobre de $c$ ?

$$
\begin{array}{ccl} 
& \text { si } c+d=10 \\
y & c & \text { es menor que } d \text { ces menor que } 5
\end{array}
$$

Figura 9. Ítem 16.

Fuente propia de la investigación.

por descuido o simplemente no es capaz de sacar conclusiones a partir de las hipótesis.

Asimismo, se concluye que la falta de comprensión de lectura en los enunciados conlleva a determinados errores. Se observó que el estudiante tiende a relacionar los enunciados de los problemas con las letras iníciales de los objetos, pero el uso de las letras como etiqueta suele conducir a errores, debido a una mala interpretación algebraica de la situación dada.

Al comparar la aplicación del instrumento en México y su réplica en Costa Rica nos permite identificar algunas regularidades y algunas diferencias.

En la investigación realizada en México, logran abordar el error con mayor profundidad al aplicar la técnica de la entrevista y esto permite explicar las causas de los errores con mayor precisión; en nuestro caso no se profundiza en las causas que inciden en el error, dejando abierta la posibilidad de ahondar más adelante.

Además, se observan también diferencias en los resultados obtenidos en la dificultad de los ítems de acuerdo con los niveles de entendimiento. Por ejemplo, el ítem $13 \mathrm{~h}$ (Figura 8) tuvo un $84.21 \%$ de respuestas correctas en nuestro caso y se ubicaría en un nivel menor a diferencia del estudio realizado en México. Es decir, en nuestro caso pasaría de un nivel III a un nivel II.

Otra diferencia se presenta en el ítem 16 (Figura 9) con un $26.32 \%$ de respuestas correctas. En este caso, el ítem obtiene un porcentaje equiparable con la dificultad presentada con las tareas del nivel IV, es decir, pasaría de un nivel III a un nivel IV.

En la figura anterior se muestra un ítem para evaluar la letra como número 
generalizado, es decir, para su solución es necesario considerar un conjunto de valores que hagan válida la expresión algebraica. Sin embargo, se obtiene que el estudiante es capaz de calcular un valor específico que hace verdadera una expresión, pero no comprende el uso de la letra como número generalizado.

Esto se considera una debilidad teórica del instrumento visto a nivel general, por tanto, es cierto que la concreción a un grupo modifica los resultados obtenidos en el análisis. Consideramos que encontrar diferencias en los niveles de utilización práctica de las letras en tareas algebraicas pone de manifiesto cierta debilidad en el instrumento utilizado. Es cierto que la validación proviene de una muestra de sujetos específica, pero su capacidad de generalización debe ser amplia. Si las respuestas a algún ítem categorizan a los sujetos en diferentes niveles, reduce sensiblemente la fiabilidad de esos ítems.

Al comparar ambos estudios es relevante mencionar que los errores encontrados son similares. El uso de la letra como objeto o etiqueta se presenta como causa de errores. También se puede señalar que cuando el estudiante se enfrenta a tareas que no sabe resolver algebraicamente, recurre a los conocimientos aritméticos y evalúa las expresiones sin razonar la respuesta dada. Se refleja una matemática memorística en el análisis de las respuestas dadas.

La falta de comprensión de lectura en los enunciados conlleva al error, es decir, el estudiante no es capaz de interpretar y razonar la información. Finalmente, se concluye que en el manejo y el uso de las letras en los distintos contextos no se obtienen los resultados esperados, pues la población participante son estudiantes universitarios y cuenta supuestamente con una formación previa en matemática.
Los ítems de mayor dificultad en ambas investigaciones responden al uso de la letra como incógnita de valor específico, letra como número generalizado o en el manejo de la letra como variable donde estas requieren un mayor nivel cognitivo.

\section{Conclusiones}

Al considerar el rendimiento académico en el curso de Matemática General se reafirma de acuerdo con los datos obtenidos. El estudiante en el nivel IV de entendimiento aprueba el curso sin ningún problema, con una nota de 92.5 sobre 100 . Sin embargo, el promedio de notas de los estudiantes que se ubican en el nivel I de entendimiento fue de 51.25 , por lo que existe una clara relación entre el rendimiento académico y el nivel de entendimiento. Se considera que el estudiante que se ubica en los niveles más bajos tiende a cometer más errores algebraicos.

Como resultado de la aplicación del instrumento se identificó una mayor complejidad en los ítems que se relacionan con el manejo de rango de valores para hacer válidas las ecuaciones algebraicas, generalizaciones de resultados, planteamiento de ecuaciones a partir de la contextualización de problemas, en la manipulación de rango de valores desconocidos, análisis de relación entre cantidades, así como en el manejo de operaciones con estructuras algebraicas abstractas.

Adu, Bossé y Chandler (2015) manifiestan que en una situación de lingüística del álgebra "los estudiantes deben interpretar la historia de portada, extraer y reducir claves información y discriminar entre información relevante e irrelevante. Esto requiere que interpretan combinaciones o configuraciones de caracteres en el registro 
verbal para discernir relaciones codificadas" (p. 19). Situación que no se evidencia como resultado de esta investigación, a los estudiantes les falta adquirir un pensamiento algebraico mucho mayor de acuerdo con el nivel educativo.

Ursini y Trigueros (2006) afirman que cuando los estudiantes logran integrar los diferentes usos de la variable y diferenciarlos es porque han alcanzado un adecuado pensamiento algebraico. Sin embargo, el concepto de variable es difícil para los estudiantes en general, los estudiantes de niveles educativos más avanzados lo utilizan con mayor flexibilidad, aunque su pensamiento algebraico en muchas ocasiones no se desarrolla como se debería.

En el presente estudio, los estudiantes se ubican mayoritariamente en el III nivel de entendimiento, es decir, son capaces de manejar las incógnitas que presentan estructuras algebraicas simples; en este nivel las letras son usadas como incógnitas de valor específico, que representan números en lugar de objetos. Además, logran hacer la reducción de términos semejantes de letras distintas dentro de la expresión algebraica.

Por otra parte, es interesante indicar que no hubo mayor diferencia entre los errores detectados por los estudiantes repitentes que participaron en el estudio y los que no. Sin embargo, se debe resaltar que sí se identificó un mayor porcentaje de estudiantes que lo cursan por primera vez en el III y IV nivel de entendimiento. Por lo tanto, se puede manifestar que el curso, como tal, no brinda las bases en el tema de álgebra, pues se parte de que este contenido debe ser adquirido previamente a la formación universitaria.

A partir de esta hipótesis no se deben presentar gran cantidad de errores, sin embargo, se evidencian vacíos conceptuales y procedimentales $\mathrm{y}$, esta podría ser una causa de los altos índices de deserción y repitencia en los cursos introductorios de matemática en el contexto costarricense a nivel universitario. Lo anterior se corrobora en el estudio realizado en el 2017 por la propia Universidad, donde se manifiesta que una de las causas de los altos índices de repitencia es la carencia de conocimientos previos (Escuela de Matemática, 2017a).

Además, Schlöglmann (2011) manifiesta que el aprendizaje de las matemáticas no solo se puede ver desde la perspectiva de la cognición, sino que los factores afectivos también tienen una fuerte influencia en el aprendizaje de las matemáticas. El nerviosismo a la hora de realizar las pruebas podría ser parte de estos factores, aspecto que ha sido señalado por Castillo, Gamboa y Hidalgo (2019) como una de las raíces del abandono y del bajo rendimiento académico en el curso Matemática General. Se podría suponer que el nervosismo puede causar en el estudiante respuestas erróneas al resolver ejercicios matemáticos.

$\mathrm{Al}$ reflexionar acerca de los errores cometidos por los estudiantes, se considera importante que el docente planifique y enseñe desde esos errores. Este es un nuevo reto que se debe asumir. Sin señalar que no posee las bases para el nivel que se cursa, el reto que se debe enfrentar como docente a nivel superior es lograr formar matemáticamente al estudiante que posee conceptos previos, así como solventar la ausencia de estos.

\section{Reconocimientos}

Esta investigación se enmarca en las actividades del programa de Máster en Didáctica de la Matemática, específicamente en el seno del Grupo de investigación "Didáctica de la Matemática: Pensamiento Numérico" de la Universidad de Granada, 
perteneciente al Plan Andaluz de Investigación, Desarrollo e Innovación de la Junta de Andalucía (FQM-193) y en el marco del proyecto de investigación EDU 201570565P "Competencia didáctica del profesor y aprendizaje de conceptos matemáticos escolares" Ministerio de Economía y Competitividad (España).

\section{Declaración de la contribución de los autores}

El porcentaje total de contribución para la conceptualización, preparación y corrección de este artículo fue el siguiente: H.B.G. 50 \% y J.L.G. $50 \%$.

\section{Declaración de disponibilidad de los datos}

Los datos que respaldan los resultados de este estudio serán puestos a disposición por el autor correspondiente H.B.G., previa solicitud razonable.

\section{Referencias}

adu-Gyamfi, K.; Bossé, M. J., \& Chandler, K. (2015). Situating student errors: linguistic-to-algebra translation errors. International Journal for Mathematics Teaching and Learning, 16(2), 1-29.

Bolaños, H. (2019) Análisis de los errores en el aprendizaje del álgebra de estudiantes universitarios (Tesis de maestría inédita). Universidad de Granada, España.

Camacho-Machín, M., Alfonso, M. C., Socas, M., \& Depool, R. (2014). University students' understanding of tasks involving ways to approximate definite integrals. Far East Journal of Mathematics Education, 13(2), 91-115. Recuperado de http://www.pphmj. com/abstract/9024.htm

Castillo-Sánchez, M., Gamboa-Araya, R., \& Hidalgo-Mora, R. (2020). Factores que influyen en la deserción y reprobación de estudiantes de un curso universitario de matemáticas. Uniciencia, 34(1), 219-245. doi: http://dx.doi. org/10.15359/ru.34-1.13

Castillo Sánchez, M., Gamboa Araya, R., \& Hidalgo Mora, R. (2018). Concordance between introductory university mathematics courses and the program of pre-university studies: a view from the perspectives of content and academic performance. Uniciencia, 32(2), 20-41. doi: http://dx.doi.org/10.15359/ru.32-2.2

Castillo-Sánchez, M., Gamboa-Araya, R. e Hidalgo-Mora, R. (2019). Caracterización y posibles causas de la deserción estudiantil en el curso de Matemática General. En Y. Morales-López (Ed.), Memorias del I Congreso Internacional de Ciencias Exactas y Naturales de la Universidad Nacional, Costa Rica, (e153, pp. 1-9). Heredia: Universidad Nacional. doi: http://dx. doi.org/10.15359/cicen.1.38

Escalante, J. E. y Cuesta, A. (2012). Dificultades para comprender el concepto de variable: Un estudio con estudiantes universitarios. Educación matemática, 24(1), 107-132. Recuperado de http:// www.scielo.org.mx/scielo.php?script=sci_arttext\&pid=S1665-58262012000100005\&ln$\mathrm{g}=\mathrm{es} \&$ tlng $=\mathrm{es}$.

Escuela de Matemática. (2017a). Informe del proyecto de investigación: La deserción y repitencia en el curso de Matemática General en la Universidad Nacional, código SIA 0250-15. Universidad Nacional. Heredia, Costa Rica: Autor.

Escuela de Matemática. (2017b). Carta al estudiante del curso de servicio MAT001 Matemática General. Universidad Nacional, Costa Rica: Autor.

García, J. Segovia, I. y Lupiáñez, J. (2014). El uso de las letras cómo fuente de errores de estudiantes universitarios en la resolución de tareas algebraicas. Boletim de Educação Matemática BOLEMA, 28(50), 1545-1566. doi: https:// dx.doi.org/10.1590/1980-4415v28n50a26

García, J. (2016). Errores y dificultades de estudiantes de primer curso universitario en la resolución de tareas algebraicas (Tesis doctoral). Universidad de Granada, España. Recuperado de http://digibug.ugr.es/handle/10481/43529

Godino, J., Castro, W., Aké, L. y Wilhelmi, M. (2012). Naturaleza del razonamiento algebraico elemental. Boletim de Educação Matemática BOLEMA, 26(42B), 483-511. Recuperado de http://www.scielo.br/pdf/bolema/ v26n42b/05.pdf 
Godino, J. y Font, V. (2003). Razonamiento algebraico y su didáctica para maestros. Granada: Universidad de Granada. Recuperado de https://www.ugr.es/ jgodino/edumat-maestros/manual/7_Algebra.pdf

Johnson, R. B. y Christensen, L. (2008). Educational Research. Thousand Oaks, California: SAGE Publications. Recuperado de https://us.sagepub.com/en-us/nam/educational-research/ book245271\#description

Küchemann, D. (1980). The understanding of generalized arithmetic (algebra) by secondary school children (Doctoral dissertation). Londres: Chelsea College, University of London.

Mulhern, G. (1989). Between the ears: making inferences about internal processes. En B. Greer y G. Mulhern (Eds.), New Directions in Mathematics Education. Londres: Routledge.

Ordoñez, O. (2014). Replicar para comprender: Prácticas investigativas para promover el razonamiento científico en estudiantes de psicología. Pensamiento Psicológico, 12(2), 7-24. Recuperado de http://www.scielo.org. co/pdf/pepsi/v12n2/v12n2a01.pdf

Pochulu, M. (2009). Análisis y categorización de errores en el aprendizaje de la matemática en alumnos que ingresan a la universidad. Colección Digital Eudoxus, 8, 1-14.

Rach, S., Ufer, S., \& Heinze, A. (2013). Learning from errors: effects of teachers training on students' attitudes towards and their individual use of errors. PNA, 8(1), 21-30. Recuperado de http://revistaseug.ugr.es/index.php/pna/ article/viewFile/6122/5440

Saglam, Y., \& Dost, S. (2016). A qualitative research on example generation capabilities of university students. International Journal of Science and Mathematics Education, 14(5), 979-996. Recuperado de https://link.springer.com/content/pdf/10.1007\%2Fs10763-015-9624-7.pdf

Schlöglmann, W. (2011). On adult unconscious beliefs in elementary algebra. En K. Kislenko (Ed.), Current state of research on mathematical beliefs XVI. Proceedings of the MAVI-16 Conference (pp. 272-287). Tallinn: Institute of Mathematics and Natural Sciences, Tallinn University.

Serres Y. (2011). Iniciación del aprendizaje del álgebra y sus consecuencias para la enseñanza. Sapiens. Revista Universitaria de Investigación, 12(1), 122-142. Recuperado de http://www.redalyc.org/articulo. oa? id=41030367007

Socas, M. (1997). Dificultades, obstáculos y errores en el aprendizaje de las matemáticas en la Educación Secundaria. En L. Rico (Coord.), La educación matemática en la enseñanza secundaria (pp. 125-154). Barcelona: Horsori. Recuperado de https://laurabrichetti.files. wordpress.com/2010/12/socas-robayna-dificutades-errores-y-obstc 3 a 1 culos-en-el-azaje-de-la-matemc3a1tica.pdf

Stravou, S. G. (2014). Common Errors and Misconceptions in Mathematical Proving by Education Undergraduates. Issues in the Undergraduate Mathematics Preparation of School Theachers, 1, 1-8. Recuperado de https:// files.eric.ed.gov/fulltext/EJ1043043.pdf

Ursini, S., Escareño, F., Montes, D. y Trigueros, M. (2005). Enseñanza del álgebra elemental: Un enfoque alternativo. México: Trillas.

Ursini, S. y Trigueros, M. (2006). ¿Mejora la comprensión del concepto de variable cuando los estudiantes cursan matemáticas avanzadas? Educación Matemática, 18(3), 5-38. Recuperado de http://www.redalyc.org/articulo. oa? $\mathrm{id}=40518302$

Vega, D., Molina, M. y Castro, E. (2012). Sentido estructural de estudiantes de bachillerato en tareas de simplificación de fracciones algebraicas que involucran igualdades notables. Revista Latinoamericana de Investigación en Matemática Educativa RELIME, 15(2), 233258. Recuperado de http://www.scielo.org. $\mathrm{mx} / \mathrm{pdf} / \mathrm{relime} / \mathrm{v} 15 \mathrm{n} 2 / \mathrm{v} 15 \mathrm{n} 2 \mathrm{a} 5 . \mathrm{pdf}$

Vergel, R. (2015). Generalización de patrones y formas de pensamiento algebraico temprano. PNA, 9(3), 193-215. Disponible en http:// funes.uniandes.edu.co/6440/1/Vergel2015PNA9(3)Generalizacion.pdf

Zamora-Araya, J. (2020). Las actitudes hacia la matemática, el desarrollo social, el nivel educativo de la madre y la autoeficacia como factores asociados al rendimiento académico en la matemática. Uniciencia, 34(1), 74-87. doi: http://dx.doi.org/10.15359/ru.34-1.5 


\section{(c) $($ ) $(9) \Theta$}

Errores en la comprensión del significado de las letras en tareas algebraicas en estudiantado universitario (Helen Bolaños-González • José Luis Lupiáñez-Gómez) in Uniciencia is protected by Attribution-NonCommercial-NoDerivs 3.0 Unported (CC BY-NC-ND 3.0) 